\title{
Retrospective assessment of inguinal hernia repair in elderly outpatients
}

\author{
P Palumbo*, A Minicucci, G Mariotta, M Girolami, A M Angelici \\ From de Senectute: Age and Health Forum \\ Catanzaro, Italy. 5-7 December 2009
}

\section{Background}

In the last ten years Day Surgery has significantly evolved, due to improvements in surgery and anaesthesia, and the need of reducing long term admission costs [1]. However, is Day Surgery suitable for elderly patients? The aim of this paper is to assess the risk of over 70 year-old outpatients who underwent inguinal hernia repair, and suggest an adequate preoperative pathway.

\section{Materials and methods}

The study analyzes collected data of a consecutive series of 211 patients submitted to groin hernia repair with open technique, under local anaesthesia. Inclusion criteria were: primary groin hernia, BMI $<30$ and good home support. All patients were preoperatively assessed on their ASA grade.

Then they were retrospectively divided in two groups, over 70 year-olds and younger. As primary outcomes mortality, postoperative main complications and unanticipated admission are considered. Secondary outcomes were postoperative pain, nausea and vomiting, wound infection, urinary retention and light bleeding.

\section{Results}

No patients reported severe complications after same day hernia repair. In the A group (62 male patients) no mortality or any other major complications were observed, despite many patients $(23-37 \%)$ having cardiac and pulmonary chronic diseases and were classified ASA III preoperatively. Only one patient had urinary retention which required catheterization. However it didn't affect the same day discharge. In the B group (147 males and 2 females) two patients had an unanticipated admission. A femoral nerve block after incorrect local anaesthesia was observed in one patient, and a further case complained of severe postoperative pain with ambulation inability. Furthermore, three patients contacted their own surgeon with minor concerns. Lastly, elderly patients referred less pain than younger ones.

\section{Conclusions}

Day Surgery in patients with inguinal hernia revealed to be safe with a very low mortality risk, even at a late age, if a careful preoperative assessment has been performed [2]. Elderly patients' preoperative pathway needs especially to assess cardiovascular and respiratory conditions. Also other diseases as diabetes, hypertension, peripheral vascular diseases and smoking need to be identified preoperatively. On the other hand, ASA III class, per se, does not represent a predictive factor for unanticipated admission, and is a less relevant factor than a careful preoperative assessment [3]. In conclusion there is no good reason to deny day surgery admission, on principle, to elderly patients undergoing inguinal hernia repair.

Published: 19 May 2010

\section{References}

1. Bettelli G: High risk patients in day surgery. Minerva Anestesiol 2009, 75:259-268.

2. Kurzer M, Kark A, Hussain ST: Day-case inguinal hernia repair in the elderly: a surgical priority. Hernia 2009, 13(2):131-136.

3. Twersky RS, Sapozhnikova S, Toure B: Risk factors associated with fasttrack ineligibility after monitored anesthesia care in ambulatory surgery patients. Anesth Analg 2008, 106(5):1421-426.

doi:10.1186/1471-2318-10-S1-A21

Cite this article as: Palumbo et al:: Retrospective assessment of inguinal hernia repair in elderly outpatients. BMC Geriatrics 2010 10(Suppl 1):A21. 